\title{
73 Required features for running shoes
}

The enormous stresses put on joints, muscles and connective tissues during locomotion demonstrate the paramount importance of proper footwear. Improper footwear can quickly cause discomfort, in the same way that a change of running shoes can work wonders for those who experience running-related pain. Unfortunately, there is no onesize-fits-all shoe that is "right" for every type of runner. Individual body weight, malpositioning of the feet or legs and incorrect foot alignment are just a few examples of why runners must always personalize their choice of footwear. Basically, a running shoe should interfere as little as possible with the foot's natural kinematic processes. Flexible soles ensure optimal rolling (heel-to-toe movement) of the shoe and thus effective force transmission from foot to ground. Stiff soles, on the other hand, force the foot into a levering motion and can cause overuse injuries because more work is transferred to the toes.

Soles and heels of running shoes should not be too thick or too wide because they intensify the effect of uneven terrain and increase the risk of twisting an ankle. Thick soles also put undue stress on the Achilles tendon because they create greater leverage forces when the foot hits the ground during laterally directed heel-to-toe movement. Lightweight footwear can force the supporting muscles to work harder in untrained individuals. Because they offer more support, firmer shoes are more appropriate for beginners.

If this "wiggle room" is missing, long periods of exercise can result in painful toenails with bruising underneath as well as the associated cramped running style. Running shoes should also feature customizable lacing. Too-loose laces are often the cause of knee pain and shin splints due to heel slippage. On the other hand, very painful pressure symptoms can occur when the lacing over the upper bridge of the foot is drawn too tight in individuals with high arches. It makes sense for runners with extensive workout routines to switch shoes regularly. This will vary, and thereby partially alleviate, the stresses exerted on joints and muscles.

The use of prescription orthopedic insoles for the acute treatment of overuse injuries should be reviewed at intervals of several weeks to several months because the insoles themselves can cause overuse injuries due to changes in foot biomechanics. It must also be remembered that the lower extremities are subject to immense stress just by normal everyday life. That is how street shoes worn every day can also cause overuse injuries. However, this fact usually escapes the attention of those afflicted because they usually do not notice the pain until greater forces are applied during exercise, leading the wearer to mistakenly blame their running shoes.

When purchasing running shoes, take into consideration that the shoe shortens during heel-to-toe movements. Therefore, the toes should have about $10 \mathrm{~mm}$ of "wiggle room" at the front. 